\title{
Effects of fetal stem cells on life quality of patients with ulcerative colitis
}

\author{
Demchuk MP , Ivankova OV ${ }^{1}$, Klunnyk MO ${ }^{1}$, Matiyashchuk IG ${ }^{1}$, Sych NS ${ }^{1}$, Sinelnyk AA ${ }^{1}$, Karayev TV ${ }^{1}$, Shulak MM1, Skalozub MV² and \\ Sorochynska KI ${ }^{3}$ \\ ${ }^{1}$ Clinical Department, Cell Therapy Centre EmCell, Kyiv, Ukraine \\ ${ }^{2}$ Laboratory and Biotechnology Department, Cell Therapy Centre EmCell, Kyiv, Ukraine \\ ${ }^{3}$ Stem Cells Bank, Cell Therapy Centre EmCell, Kyiv, Ukraine
}

\begin{abstract}
In this article we briefly summarized on the main characteristic features of ulcerative colitis (UC) and accentuated on reduced life quality among the patients with UC, in particular, effect of clinical manifestations of the disease on everyday life was described in such patients.

Unambiguously, the principal direction to better life quality in patients with UC consists in decreasing activity of inflammatory process. Significant aspect in solution of this problem for present day suggests use of fetal stem cells (FSCs) in complex treatment of the patients suffering from UC. Specifically, by virtue of stem cell therapy use, rapid lowering of clinical signs of UC was recorded, patients revealed stabilization of hemoglobin and total protein levels in blood. Changes of such parameters in the CG patients tended to be much slower.

Within the whole period under study, we recorded a consistent pattern between decreased evidence of underlying disease manifestation that is coupled with higher life quality among the UC patients. In conformity with IBDQ international questionnaire, describing that bowel symptoms, systemic symptoms, emotional and social function, life quality among UC patients of the MG were increased (51.33\% vs. 96.43\%), whereas the patients of the CG reported the value $61.16 \%$ that was higher only within the range of $86.16 \%$.
\end{abstract}

Administration of suspensions with FCSs for complex therapy in UC patients proved to be one of the ways for refining social and psychological wellbeing of the individuals under study.

\section{Introduction}

Ulcerative colitis (UC) is a chronic inflammatory disease of the wall of large intestine, mostly affecting proximal region with ulcerous necrotic changes of intestinal wall characterized by recurrent, progressive course that can be clinically presented as intermittent loose stool with bleeding being infrequently complicated by rectal bleeding, perforation, bowels stenosis and sepsis.

Insufficiency of immune regulation results in uncontrolled response to different agents; and, simultaneously, it contributes to local damage of tissues by associated inflammatory process. In addition, a likely development of inflammatory reactions causes impairment of barrier functions throughout the intestines, mucus formation, metabolic and deregulatory disturbances along with chronic intoxication. UC belongs to autoimmune diseases which owing to affected immune response cannot stop acute inflammation and leads to a chronic process development. UC is regarded as multi-factor disease with genetic predisposition [1].

$\mathrm{UC}$ is a disease lasting during the whole life and refers to complicated social and psychological well-being of human [2]. According to the data of WHO, quality of life is an integral assessment in the individual in respect to his/her position in the society, within system of common human values as well as relation between person's condition and fixed goals with all potentialities. Quality of life is a grade of both inner comforts of the human and likely community estimates by the society people can normally live in. The patterns of UC symptoms have different effects on reduced activity of daily living among the patients suffering from this disease.

Thus, stool frequency, doughy stool with blood, pus and mucus can create a prominent discomfort for the patients while staying outside the house. UC patient constantly thinks about the rest room because of tenesmus and feeling of defecation need might happen at the most inexpedient moment. All the symptoms above interfere with concentration on one's professional duties, may result in a range of inconveniences for leisure-time activity, could hinder a possibility of doing favourite sports etc. Likely problems promote nervousness and irritability of the patients, which inevitably lead to severe psychological obstacles, quite often demanding a specific psychology care for the patients.

Diarrhoea contributes to dehydration, various disruptions of blood electrolytes resulting in a marked general weakness and body weight reduction among patients. Abdominal cramps growing much intense

Correspondence to: MP Demchuk, Internal Medicine, Cell Therapy Center EmCell; 37 A Syretska Street, Kyiv City, 04073, Ukraine, Tel: +380-688-898-989; E-mail: mdmaryap2017@gmail.com

Key words: Fetal stem cells, IBDQ international questionnaire, life quality, ulcerative colitis

Received: February 21, 2018; Accepted: March 14, 2018; Published: March 20 2018 
prior to defecation and in some of the patients pains tend to gradually increase over 30-90 minutes following food ingestion that requires regular administration of medicines to decrease particular symptoms. Constant enforced use of analgesics and spasmolytic drugs favours unwanted symptoms as related to likely adverse reactions in the UC patient. To escape severe symptoms, the patients can deliberately refuse from taking foods; especially, if they stay out-of-doors and this promotes general patient's exhaustion, decreased body weight various problems, straight to chronic illness cachexia. Regular deficit of vitamins, microelements, proteins and the other vitally important food supplements for the patients (usually absorbed in gastrointestinal tract) can result in hair loss, worsening their skin condition, disrupted endocrine functions etc. - all this becomes a favourable base for development of relevant astheno-hypochondriac and depressive syndromes.

In case of extra-intestinal lesions, typical symptoms of UC also involve some traces of affection over the other organs. The most remarkable are skin damage with gangrenous pyoderma or erythema nodosum; lesions of the joints with sacroiliitis or peripheral arthritis; canker sore, sore eyes (episcleritis, uveitis, iridocyclitis), sclerosing cholangitis, as well as different bone lesions (commonly resulting from therapy using steroids) - e.g. osteoporosis, bone softening or aseptic necrosis.

Problems with locomotor apparatus can restrict mobility in UC patient that makes the person search for some occupation non-related to persistent physical loads. Frequently such patients can change their profession because of disease that reflects a negative psychology sequel owing to inability to get engaged into favourite business, financial expenses related to lower salary etc. Such patients need to be kept off travelling and doing sports; this can pull them away from their friends - again, all the above can emphasize inability of such patients to benefit from their lives worth living.

Canker sore can impair normal nutrition among UC patients and additionally leads to refusal from food and weight loss among such patients.

Sore eyes (episcleritis, uveitis, and iridocyclitis) cause reduced vision acuity making some restrictions both for the choice of career and organization of patient's external environment.

Skin lesions also interfere with normal everyday life enforcing the patient to stay at home, demanding from the individual to follow additional hygienic procedure with topical treatment; ultimately, can create a cosmetology defect in the patient.

Ongoing and recurrent course of UC along with progressive damage to the structure and functions of intestinal mucosa is associated with significant decrease of life quality among the patients; raising chronic intestinal diseases to the extent of essential medical-social problems and suggests searching for the contemporary and new ways to study disease aetiology and pathogenesis as well as enhancing prevention and treatment of this problem. One of the directions to higher life quality among the patients with UC is much concentrated on application of foetal stem cells (FSCs) in complex treatment [3-6].

\section{Material and methods}

Within a long period of time 32 men with confirmed diagnosis of UC were under study at Cell Therapy Centre EmCell including 21 patients allocated to the main group (MG) and 11 patients were assessed in the control group (CG). Diagnosis verification in patients of both groups was made on the base of clinical manifestations, disease history, results of physical, laboratory and instrumental examinations. Active pathology process at the moment of stem cell treatment was regarded as the main criterion of choice for the patients to allocate to the above study. Individuals undergoing surgery treatment on the underlying disease were not included into the MG. Age category of patients with UC in the MG made up the persons from 18 to 60 years, whereas patients in their age range from 18 to 55 years were studied in the CG. Distribution of the patients by sex was practically similar in both groups: 10 men and 11 women were referred to the MG, whereas CG patients were split to 6 women and 5 men. Disease duration among the patients of both groups composed more than 5 years.

With consideration of all risks related to endoscopic and roentgenology manipulations on the patients with UC disease in acute phase owing to a high expectation of intestinal perforation, we pivoted from a mandatory performing such procedures prior to FSCs transplantation. Doctors mainly concentrated on analysing the main clinical symptoms as well as control of laboratory parameters in progression. The extent of clinical symptoms expression was evaluated in scores as follows: 0 - none, $1+$ - slightly expressed, $2+-$ marked, 3+ - significantly expressed.

Patient's venous blood was tested to evaluate haemoglobin levels by use of automated blood analyser and application of photometric technique; whereas erythrocytes sedimentation rate (ESR) was assessed by Westergren method for ESR and total protein ranges were studied by means of colorimetric method.

Estimation of life quality among the patients with UC was performed by means of Inflammatory Bowel Disease Questionnaire (IBDQ) scale. This questionnaire suggests 32 questions within four sections. It allows general evaluation along with assessment of intestinal, systemic, social and emotional manifestations of UC disease. A range of the scores on provision of giving the answers to each of the question was from 1 to 7 . Minimal total value constituted 32 scores in accordance with the results of the questionnaire, whereas maximum value was 224 . The highest score corresponds to the best parameters of life quality among the patients under study.

The main goal of treatment was reduction of clinical signs of UC and increasing life quality among the patients. We used a complex approach to treatment of UC where, in addition to standard therapy by drugs, FSCs treatment as an efficient contemporary method for stem cell transplantation was administered for the patients during a protracted period of time, according to the prescriptions made from their attending doctors.

All patients of the MG underwent one course of treatment using FSCs [7]. Foetal stem cells were extracted from 5-12 weeks gestation foetuses [8]. Foetuses were procured from healthy women, following induced interruption of pregnancy in accordance with social indications and performed in medical institutions. All such women underwent a preliminary testing for viral and hemic diseases. All works with foetal material were conducted in conformity with current legal and ethical standards in Ukraine [9-11].

Prior to stem cell transplantation, all patients got verbally acquainted with the plan of treatment and all procedures for diagnosismaking. Later all patients were informed in respect to the results of clinical, laboratory and instrumental investigations. The patients were also well-versed regarding the potential of FSCs suspensions as well as the method of stem cell transplantation. After clarifying on the 
treatment plan and following description of the procedure, the patients signed their written informed consent.

Immediately after withdrawal out of stem cells bank (cell suspensions are cryopreserved in liquid nitrogen at minimal temperature of $196^{\circ} \mathrm{C}$ ), FSCs suspensions had been individually selected for each patient. For clinical use, all cryopreserved FSCs were controlled for bacterial and viral infections (Treponema pallidum, Toxoplasma gondii, Chlamidia trachomatis, Mycoplasma hominis, Mycoplasma genitalium, Ureaplasma Parvum, Urealyticum, HIV-1, HIV-2, HBV, HCV, HGV, HPV, CMV, EBV, HHV6, HSV-1,2, Parvovirus B19). All suspensions had a definite number of nucleated cells and CD34+, range of colony forming units and definite cell viability prior to suspension cryopreservation.

Defrost of the suspension was carried out in accordance with standard protocols. The containers were evacuated from liquid nitrogen immediately prior to FSCs administration; later cells were immersed into a water bath for thawing at $+37^{\circ} \mathrm{C}$ and kept there until a liquid phase. All further procedures were performed at indoor temperature in accordance with a strict compliance to the aseptic rules. Additional control upon suspension quality, microscopic studies and, specifically, calculation of viable cells were conducted prior to FSCs administration: by means of the counting chamber microscopic trypan blue staining method was applied along with the flow cytofluorometry method use.

Our treatment suggested intravenous drip-feed infusion of suspension containing FSCs extracted from human foetal liver on top of $200 \mathrm{~mL} 0.9 \%$ saline solution after previous intravenous jet i.v. premedication using $10 \mathrm{mg}$ dimedroli and $30 \mathrm{mg}$ prednisolone. Speed of solution administration made up 20-40 drops per minute. The amount of administered suspension was from $3.0 \mathrm{~mL}$ up to $5.0 \mathrm{~mL}$ per one course of treatment. The number of nucleated cells ranged from 9 to $35 \times 10^{6} / \mathrm{mL}$, forasmuch as percentage of viable cells - was not less than $70 \%$.

During the next 2 days FSCs suspensions containing hematopoietic progenitor cells of spleen and cells extracted from soft tissues were administered - tend to create a depot within adipocellular tissue in the patient.

Preparation in amount from 3.5 to $6.5 \mathrm{~mL}$ was injected per each course of treatment. Patients of both groups were given preparations including 5-aminosalicylic acid as the basic therapy in daily dose of 3-4 g.

Dynamics of the results acquired in accordance with the principal clinical symptoms (abdominal cramps, tenesmus (tender feeling of defecation need), feeling of false defecation need, nocturnal stool, rectal bleeding, mucous admixtures in stool, stool frequency, heart rate, body temperature, extra-intestinal symptoms (asthenoneurotic syndrome, arthralgia) as well as laboratory findings (haemoglobin, total protein, ESR) in the selected periods under observation (prior to treatment, over 15, 30, 90, 180, 270 and 360 days after therapy) both for the MG and CG patients have been suggested in the Tables 1,2, 3 and 4 .

Statistical processing of the results received was made with the help of computer software program Statistica.

\section{Results and discussion}

Thirty-two patients with clinical cases of UC have been under study. FSCs were used in complex treatment for 21 patients, whereas the rest 11 patients suffering from UC underwent their conventional therapy. Before administration of FSCs to the patients, depending on disease localization, 5 patients of the MG revealed an inflammatory process presented as proctitis, and it was also diagnosed in 5 patients of the CG, whereas 12 patients of the MG along with 6 patients in the CG were presented with left-side colitis respectively. Simultaneously, total colitis was recorded in 4 patients of the MG. Loss of body weight was remarkable for the patients of both groups and such ranges were from 5 to $15 \mathrm{~kg}$. During the first 3 days following FSCs administration, all patients of the MG reported a syndrome of early post-infusion improvements associated with a fit of energy, better appetite, higher physical and mental activity. In addition, these patients also revealed their optimistic judgement. We did not observe a rapid growth of positive effects in treatment among the patients of the CG. Three patients of the CG over the 4th day under study were additionally administered steroids $30 \mathrm{mg}$ and peroral prednisolone within a period of 5 days, owing to a significant disease activity reported by them. Patients in both groups were presented with a significant expression of associated pain (3+). No pain was recorded in patients of the MG over day 90 under study, whereas UC patients in the CG were presented with minor expression of pain. A tender feeling of defecation need (tenesmus) was not detected in the MG patients over day 30 under study, whereas in the CG the same manifestations still remained as non-significant until day 90 under study. False feeling of defecation need over 30 days following treatment in both groups was not significant and we did not record them throughout the other periods of observation. Nocturnal feeling of defecation need was characteristic for UC patients in both groups during the whole period under study. Evidence of rectal bleeding presented with bloody stool was recorded in the patients of both groups up to day 90 since we began a follow up. Mucous admixtures in stool were remarkable for the whole study period in the CG patients, forasmuch as likely signs were not recorded in the MG patients. Body temperature drop to $36.6^{\circ} \mathrm{C}$ in the MG patients had been already reported by the day 15 under study. Beginning from day 90 under observation, the patients of both groups did not complain of body temperature increasing higher than $36.8^{\circ} \mathrm{C}$. At the baseline the signs of asthenoneurotic syndrome were characteristic for all the patients. Until the end of study period such a syndrome was remarkable for 9 patients $(42.86 \%)$ in the MG and was revealed in 6 patients of the CG (54.55\%). Arthralgia episodes were reported by 1 patient in the MG and 1 patient of the CG was presented with likely complaint until the end of period under study.

Patients in both groups had mild anaemia before treatment. Baseline haemoglobin levels in the MG made up $101.3 \pm 3.3 \mathrm{~g} / \mathrm{L}$ and patients of the CG recorded similar ranges at $109.3 \pm 2.6 \mathrm{~g} / \mathrm{L}$. Stabilization of haemoglobin levels was observed by day 90 and it constituted $125.9 \pm 3.6 \mathrm{~g} / \mathrm{L}$ in the MG and $120.7 \pm 3.3 \mathrm{~g} / \mathrm{L}$ for the CG patients. It is noteworthy that patients in the MG demonstrated growth of haemoglobin by 24.6 points, whereas within the same period the patients of the CG revealed haemoglobin level which remained higher approximately by 11.4 score. Until the end of observation period the levels of peripheral blood haemoglobin reached their stabilization among the patients of both groups.

Total protein level was reduced in blood of patients in both groups and this value made up $56.2 \pm 8.3 \mathrm{~g} / \mathrm{L}$ in the MG and $60.5 \pm 6.3 \mathrm{~g} / \mathrm{L}$ for the CG patients. Stabilization of ranges of protein in the MG was observed by day 90 under study and it composed $-68.8 \pm 8.7 \mathrm{~g} / \mathrm{L}$, in respect that by the day 180 the CG patients recorded such levels by 65.6 $\pm 8.7 \mathrm{~g} / \mathrm{L}$.

At the baseline, ESR elevated by $24.0 \%$ within the upper limit of normal, whereas the patients of the CG revealed likely increasing by $18.3 \%$. Stabilization of ESR had been just recorded starting from the period of 90 days. 
Based on all evidence presented above, one can summarize on a positive influence of FSCs upon the course of UC among the patients. As it had been proved, the patients of the MG undergoing complex treatment using FSCs were presented with the main clinical pattern of UC was descending faster; simultaneously, the levels of blood haemoglobin and total protein in such individuals had been also stabilized. Changes in these values tended to be particularly slower among the patients of the CG.

Dynamics of the main clinical symptoms along with the basic laboratory findings are presented in the Tables 1-4.

Assessment of life quality among the patients with UC (both for the MG and CG) has been presented in the figure 1: Inflammatory Bowel Disease Questionnaire (Figure 1). The first 2 columns of this diagram correspond to dynamics of "bowel symptoms" for different periods under study. In conformity with 7 points scale we evaluated the parameters for the extent of expression of bowel symptoms as followed: "stool frequency", "loose stool", "abdominal cramps", "passing gas", "abdominal bloating", "rectal bleeding", "feeling of defecation need", "soiling", "sick stomach" etc. Such values might specifically affect the rate of satisfaction with life among the patients with UC. The baseline value of "bowel symptoms" in both groups corresponded to a marked discontent among the patients and made up 34 points in the MG and 36 in the CG out of minimal 10 scores. Over 30 days under study, the number of scores increased by 50 in the MG patients, in respect that CG patients revealed increasing of the same score by 45 . Over 90 days under study, the rate of satisfaction with life in the MG patients constituted 63 points and 54 scores for the CG. At the end point of observation over 360 days such values were recorded as 67 scores (MG) and 59 scores (CG) out of maximum 70 points.

The next columns 3 and 4 on the diagram reflect "systemic symptoms", which summarize such manifestations as: "tired and worn out", "energy", "generally unwell", "sleeping disturbance", "weight problems". At the baseline of observation among the patients of the MG discontent with their life made up 17 points, whereas the same value in the CG was 20 scores out of minimal 5 points. Significant reduction of likely discontent was remarkable for the period of observation by 90 days and constituted 33 points in the MG and 29 points in the CG patients. Maximum life satisfaction was recorded for the period of observation by 180 days and it remained stable until the end of the study period making up 34 scores in the MG and 31 points out of maximum 35 points among the patients of the CG.

Life quality evaluated by the components of emotional well-being at the baseline was the highest over the other indexes by similar scale -49 points for the MG patients and 63 points out of minimal 12 points in the CG. Emotional function consists of the next components: "frustrated/ impatient", "worried about surgery", "fear of not finding a toilet", "depressed/discouraged", "disease-related worries", "relaxed/free of tension", "embarrassed with bowel movement", "tearful or upset", "angry owing to bowel symptoms", "irritable, lack of understanding", "satisfaction with personal life". For the 30th day under study quality of life in the patients of the MG elevated by 21 points, in respect that the same value of the CG was higher by 6 points and constituted 70 versus 68 scores respectively. At the end point under observation by 360 days - life quality in emotional sphere of UC patients reached 82 points in the MG and 73 points in the CG out of maximum 84 scores.

Quality of live in patients suffering from UC in the social sphere was rather low and made up 15 scores in the MG patients and 19 points among the patients of the CG (out of maximum 5 scores). Social function was assessed by the following criteria: "work/school", "cancel social engagement", "leisure or sports activities", "avoid events without toilets", "sexual activity". Nevertheless, already after day 30 under study the MG patients revealed life quality in social aspect which was twofold increased, whereas the same parameter remained 6 points higher in the CG and constituted 30 versus 25 points respectively. Maximal discontent in social sphere among the patients of both groups was recorded at the end of observational period by 360 days and made up 33 scores in the MG and 30 scores in the CG out of maximum 35 points.

Inflammatory Bowel Disease Questionnary

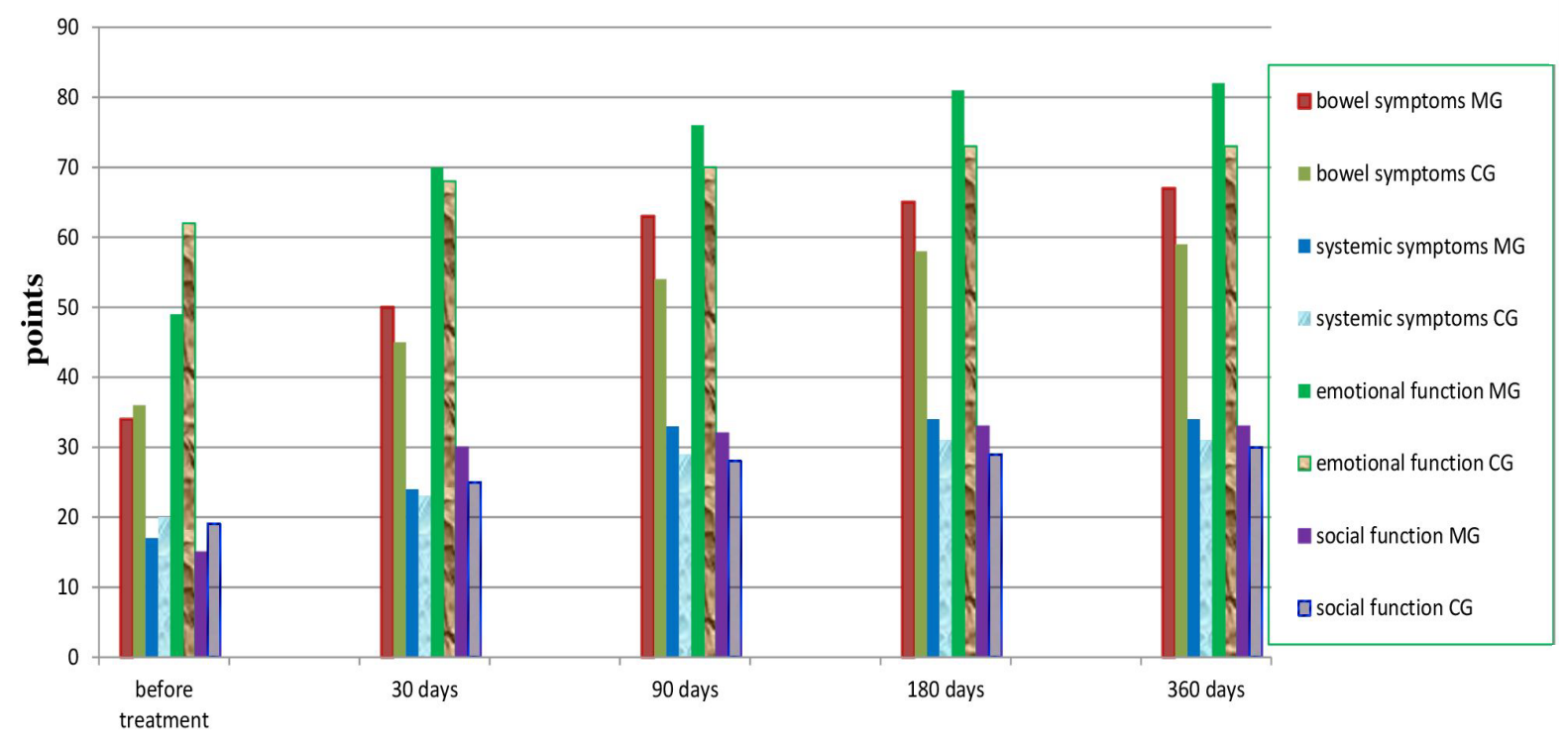

period of observation

Figure 1. Inflammatory Bowel Disease Questionnaire (IBDQ)

Legend : MG - main group; CG - control group 
Table 1. Dynamics of the main clinical symptoms among the patients of the MG

\begin{tabular}{|c|c|c|c|c|c|c|c|}
\hline $\begin{array}{l}\text { Clinical symptoms } \\
\text { (MG) }\end{array}$ & Before treatment & Over 15 days & Over 30 days & Over 90 days & Over 180 days & Over 270 days & Over 360 days \\
\hline $\mathrm{n}-21$ & \multicolumn{7}{|c|}{ Evaluation in scores } \\
\hline Abdominal cramps & $3+$ & $2+$ & $1+$ & 0 & 0 & 0 & 0 \\
\hline $\begin{array}{l}\text { tender feeling of defecation need } \\
\text { (tenesmus) }\end{array}$ & $3+$ & $1+$ & 0 & 0 & 0 & 0 & 0 \\
\hline False feeling of defecation need & $3+$ & $1+$ & $1+$ & 0 & 0 & 0 & 0 \\
\hline Nocturnal stool & $2+$ & $2+$ & $2+$ & $1+$ & $1+$ & $1+$ & $1+$ \\
\hline Blood in stool & $3+$ & $2+$ & $1+$ & $1+$ & 0 & 0 & 0 \\
\hline \multirow[t]{2}{*}{ Mucous admixtures in stool } & $3+$ & $2+$ & $1+$ & $1+$ & $1+$ & $1+$ & $1+$ \\
\hline & \multicolumn{7}{|c|}{ Values (min-max) } \\
\hline stool frequency, (number) & $3-30$ & $3-16$ & $3-8$ & $2-6$ & $2-4$ & $2-4$ & $2-4$ \\
\hline HR per minute & $86-98$ & $86-92$ & $82-90$ & $78-86$ & $72-84$ & $70-84$ & $70-84$ \\
\hline \multirow[t]{2}{*}{ Body temperature, ${ }^{\circ} \mathrm{C}$} & $37,2-37,7$ & $36,6-37,1$ & $36,6-37,1$ & $\leq 36,8$ & $\leq 36,8$ & $\leq 36,8$ & $\leq 36,8$ \\
\hline & \multicolumn{7}{|c|}{ \pm number } \\
\hline Asthenoneurotic syndrome & 21 & 17 & 13 & 11 & 11 & 9 & 9 \\
\hline Arthralgia & 7 & 5 & 3 & 2 & 2 & 1 & 1 \\
\hline
\end{tabular}

MG: Main Group; HR: Heart Rate

Table 2. Dynamics of the main clinical symptoms among the patients of the CG

\begin{tabular}{|c|c|c|c|c|c|c|c|}
\hline $\begin{array}{l}\text { Clinical symptoms } \\
\text { (CG) }\end{array}$ & Before treatment & Over 15 days & Over 30 days & Over 90 days & Over 180 days & Over 270 days & Over 360 days \\
\hline$n-21$ & \multicolumn{7}{|c|}{ Evaluation in scores } \\
\hline Abdominal cramps & $3+$ & $2+$ & $1+$ & $1+$ & 0 & 0 & 0 \\
\hline $\begin{array}{l}\text { tender feeling of defecation need } \\
\text { (tenesmus) }\end{array}$ & $3+$ & $2+$ & $1+$ & $1+$ & 0 & 0 & 0 \\
\hline False feeling of defecation need & $3+$ & $2+$ & $1+$ & 0 & 0 & 0 & 0 \\
\hline Nocturnal stool & $2+$ & $2+$ & $2+$ & $1+$ & $1+$ & $1+$ & $1+$ \\
\hline Blood in stool & $3+$ & $2+$ & $1+$ & $1+$ & 0 & 0 & 0 \\
\hline \multirow[t]{2}{*}{ Mucous admixtures in stool } & $3+$ & $2+$ & $2+$ & $1+$ & $1+$ & $1+$ & $1+$ \\
\hline & \multicolumn{7}{|c|}{ Values (min-max) } \\
\hline stool frequency, (number) & $3-25$ & $3-18$ & $3-8$ & $2-8$ & $2-8$ & $2-6$ & $2-6$ \\
\hline HR per minute & $82-90$ & $86-92$ & $82-90$ & $76-86$ & $72-82$ & $70-82$ & $70-82$ \\
\hline \multirow[t]{2}{*}{ Body temperature, ${ }^{\circ} \mathrm{C}$} & $37,1-37,7$ & $37,1-37,4$ & $36,9-37,1$ & $\leq 36,8$ & $\leq 36,8$ & $\leq 36,8$ & $\leq 36,8$ \\
\hline & \multicolumn{7}{|c|}{ \pm number } \\
\hline Asthenoneurotic syndrome & 11 & 11 & 11 & 8 & 6 & 6 & 6 \\
\hline Arthralgia & 8 & 8 & 6 & 6 & 2 & 1 & 1 \\
\hline
\end{tabular}

CG: Control Group; HR: Heart Rate

Table 3. Dynamics of the principal laboratory findings among the patients of the MG

\begin{tabular}{|c|c|c|c|c|c|c|c|c|}
\hline $\begin{array}{c}\text { Laboratory } \\
\text { findings (MG) }\end{array}$ & $\mathbf{R R}$ & Before treatment & Over 15 days & Over 30 days & Over 90 days & Over 180 days & Over 270 days & Over 360 days \\
\hline \multicolumn{9}{|l|}{$n-21$} \\
\hline hemoglobin, $\mathrm{g} / \mathrm{L}$ & $117-160$ & $101.26 \pm 3.29$ & $110.27 \pm 3.7$ & $114.22 \pm 3.65$ & $125.87 \pm 3.64$ & $130.34 \pm 4.3$ & $131.23 \pm 3.7$ & $132.15 \pm 3.9$ \\
\hline Total protein, $\mathrm{g} / \mathrm{L}$ & $65-85$ & $56.2 \pm 8.3$ & $58.1 \pm 9.3$ & $58.8 \pm 8.7$ & $68.8 \pm 8.7$ & $72.8 \pm 11.7$ & $73.8 \pm 10.7$ & $74.9 \pm 8.5$ \\
\hline $\mathrm{ESR}, \mathrm{mm} / \mathrm{H}$ & $1-30$ & $37.2 \pm 2.3$ & $35.3 \pm 3.5$ & $33.2 \pm 3.3$ & $27.2 \pm 2.7$ & $25.2 \pm 2.3$ & $17.9 \pm 4.3$ & $23.2 \pm 2.9$ \\
\hline
\end{tabular}

MG: Main Group; ESR: Erythrocytes Sedimentation Rate

Table 4. Dynamics of the principal laboratory findings among the patients of the CG

\begin{tabular}{|c|c|c|c|c|c|c|c|c|}
\hline $\begin{array}{c}\text { Laboratory } \\
\text { findings (CG) }\end{array}$ & $\mathbf{R R}$ & Before treatment & Over 15 days & Over 30 days & Over 90 days & Over 180 days & Over 270 days & Over 360 days \\
\hline \multicolumn{9}{|l|}{$\mathrm{n}-11$} \\
\hline hemoglobin, $\mathrm{g} / \mathrm{L}$ & $117-160$ & $109.3 \pm 2.6$ & $111.3 \pm 3.8$ & $112.3 \pm 3.5$ & $120.7 \pm 3.3$ & $125.4 \pm 3.7$ & $126.2 \pm 4.1$ & $125.5 \pm 3.2$ \\
\hline Total protein, $\mathrm{g} / \mathrm{L}$ & $65-85$ & $60.5 \pm 6.3$ & $61.1 \pm 7.4$ & $62.2 \pm 9.7$ & $63.8 \pm 7.6$ & $65.6 \pm 8.7$ & $67.8 \pm 9.3$ & $68.2 \pm 9.8$ \\
\hline $\mathrm{ESR}, \mathrm{mm} / \mathrm{H}$ & $1-30$ & $35.5 \pm 3.3$ & $34.3 \pm 3.5$ & $33.7 \pm 4.3$ & $30.2 \pm 3.5$ & $28.2 \pm 3.3$ & $25.9 \pm 5.7$ & $25.4 \pm 3.8$ \\
\hline
\end{tabular}

CG: Control Group; ESR: Erythrocytes Sedimentation Rate 
In the whole, after evaluation by Inflammatory Bowel Disease Questionnaire one can summarize that baseline life quality of the MG patients reduced by $48.67 \%$, forasmuch as the same value in the CG was lower by $38.84 \%$, which is tightly correlated with the clinical signs of the disease. Within the period under study we observed a trend when life quality of UC patients elevated along with decrease in expression of clinical pattern on underlying disease at the equal basis with reduction in disease activity. Thus, over 90 days quality of life in patients of the MG increased by $39.74 \%$, whereas patients of the CG recorded likely elevation by $19.64 \%$. Better and more rapid results we managed to get among the MG patients who were administered FSCs preparations as a complex treatment despite of worse baseline clinical and laboratory parameters if compared to the patients of the CG. At final of the period under study quality of live in the MG patients was lower only by $3.57 \%$, likewise in the CG patients it was reduced by $13.84 \%$. It is worthy, and we shall consider that in all MG patients UC disease remission had been achieved even without use of corticosteroids.

\section{Conclusion}

Use of FSCs preparations in complex treatment of the patients with UC is one of the modes to enhance life quality of such patients. In particular, such results are facilitated by the main clinical effects among the patients with likely eradication (decreasing) the symptoms of underlying disease presented as: stabilization of stool; disappearance of cramps, tenesmus; temperature stabilization and lower rates of disease severity, elimination of anaemia signs in the patients and inhibition of weight loss along with body mass stabilization in such patients. Lower frequency of extra-intestinal manifestations of ulcerative colitis, as well as positive changes in psycho-functional and emotional spheres, cut down on all indications for necessary surgery interventions on UC patients.

\section{Author Contributions}

All authors of the manuscript contributed much to this scientific study and performed the following research activity:

M.P. Demchuk - development of study concept and design, statistic processing of the results of investigation, analysis of acquired data and text preparing.

O. V. Ivankova - conduction of study, statistic processing of the results of investigation, and analysis of received results

M.O. Klunnyk - conduction of study, statistic processing of the results of investigation, and analysis of received results

I.G. Matiyashchuk - conduction of study, analysis of acquired data

N.S. Sych - conduction of study, analysis of received results
A.A. Sinelnyk - conduction of study, analysis of acquired data

T.V. Karayev - conduction of study, analysis of received results

M.M. Shulak - conduction of study, analysis of acquired data

M. V. Skalozub - performance of laboratory tests and analysis of findings

K.I. Sorochynska, - responsibility for fetal stem cells

\section{Acknowledgments}

All authors made a great contribution to statistic data processing and conduction of all studies on the patients with ulcerative colitis and complex treatment by use of fetal stem cells.

\section{Competing interests}

All authors emphasize absence of financial and other conflict interests in respect of the submitted manuscript. There are no matters relevant to the conflict of interests among the authors who contributed to manuscript submission. All works with foetal material were conducted in accordance with current Ukrainian legal and ethical standards.

\section{References}

1. Gandzha SM, Kovalenko VM, Shuba NM et al. 2002 Internal diseases. K.: Zdorovya. P. 992

2. Stenke E, Hussey S (2013) Ulcerative colitis (CG166), Management in adults, children and young people. NICE (UK). Arch Dis Child Educ Pract Ed. 99: 194-7. [Crossref]

3. Bushnyeva VA (2003) Transplantation of Hemopoietic Stem Cells Suspensions in Complex Treatment of Ulcerative Colitis in patients with nonspecific ulcerative colitis // Vinnitsa State Medical University Bulletin 1/1: P.162.

4. Smikodub AI, Bushnyeva VA. (2003) Transplantation of Hemopoietic Fetal Stem Cells in Complex Treatment of Ulcerative Colitis // Naval Medical Bulletin. Materials of International scientific-practical conference "Sustainable and disputable problems of surgery on hepato-pancreatic-duodenal biliary zone"

5. Smikodub AI, Bushnyeva VA. (2004) Management of patients with nonspecific ulcerative colitis and Crohn's disease in practice of family doctor // Family medicine. 3: P. 139-141.

6. Smikodub AI, Bushneva VA. (2003) Transplantation of Hemopoietic Fetal Stem Cells in Complex Treatment of Ulcerative Colitis // Naval Medical Bulletin. 2. P. 287-291.

7. Method of treatment of people with embryonic cell suspensions / Smikodub O.I 15.03.2005 Patent 64826 Ukraine.

8. Harvesting of hematopoietic stem cells from human embryonic hematopoietic organs / Smikodub O.I., Novytska A.V., Markov I.S. 17.09.2001 Patent 41302 Ukraine.

9. Law of Ukraine about organ transplantation and other anatomic materials to the person

10. Ethical issues and norms at work with human embryonic tissues: Method Recommendations. Authors: Smikodub OI, Arkhipenko IV, Bushnyeva VO, Demchuk MP, Novytska AV, Radziyevska LV, Samus NV, Smikodub OO - Kyiv, - 2004. - P. 20.

11. The Civil Code of Ukraine, article 290.

Copyright: (C2018 Demchuk MP. This is an open-access article distributed under the terms of the Creative Commons Attribution License, which permits unrestricted use, distribution, and reproduction in any medium, provided the original author and source are credited. 